\title{
Time for a Standardized Common Femoral Artery Classification System
}

\author{
Thomas Zeller ${ }^{1}$
}

Received: 21 November 2021 / Accepted: 25 November 2021/Published online: 15 February 2022

(C) The Author(s) 2022

During the last decade, interventional therapy of common femoral artery (CFA) lesions has become an attractive alternative to the open surgical reconstruction by means of endarterectomy with or without patch plastic. However, prospective comparative data between endovascular treatment and surgery are still rare mainly comparing stenting with TEA [1]. CFA lesions may differ regarding location (exclusive CFA involvement vs. extension into the femoral bifurcation or external iliac artery) and morphology (e.g., degree of calcification). Therefore, there is a need for a standardized classification system to allow the comparison of published data as well as to identify treatment strategies that fit best individual lesion characteristics.

The modified coronary Medina classification proposed by Bonvini et al. is limited by considering only the anatomical lesion location and lesion extension [2]. The Azema classification included information about lesion morphology defining four types of lesion locations [3] but the degree of calcification and the severity of the lesion (stenosis vs. occlusion) were not part of the classification system. However, these missing parameters are potential predictors for acute treatment success and durability of the revascularization procedure.

In the present study, Rabellino et al. modified the Azema classification in a very practical way [4]: Previous Type IV lesions defined as bypass stenotic lesions were excluded in order to limit the new classification to native artery atherosclerotic lesions. Hence, Type I-III lesions

Thomas Zeller

Thomas.zeller@uniklinikum-freiburg.de

1 Clinic for Cardiology and Angiology II, University Heart Center Freiburg - Bad Krozingen, Campus Bad Krozingen, Südring 15, 79189 Bad Krozingen, Germany remained the same. Type IV lesions in the new classification include lesions extending either from the external iliac artery (EIA) or common iliac artery (CIA) into the CFA and affecting its bifurcation.

Three additional lesion sub-characteristics were added for a more detailed lesion description of the four types of lesions: In Type III and IV lesions, it was specified which branch of the femoral bifurcation is involved. If only the superficial femoral artery (SFA) is affected, this was classified with an "S." If only the deep femoral artery (DFA) was involved, a " $D$ " was given, and if both branches were involved, it was classified as a "B" for both. As such, a Type III lesion starting at the CFA and extending to both the branches was denominated as a "TYPE III B" lesion. A further differentiation involves stenotic ("S") versus occlusive ("O") lesions. Finally, vascular calcium assessment was made by fluoroscopy or by computed tomography and added to the classification in heavy calcium burden " $\mathrm{H}$ " or with mild to moderate calcium burden "M." Back to the example of a Type III B lesion with occlusive disease and heavy calcium burden, the lesion would be classified as a Type III B, O, H lesion.

Classifying CFA lesions in this proposed fashion in upcoming studies will allow a better comparison of acute and potentially long-term outcomes. Besides the small sample size, the main limitation of the study is the unusual definition of primary patency ("time free from more than $50 \%$ restenosis following index treatment"). This makes it impossible to compare the primary study end point of the Rabellino with historic studies and limits the prediction of long-term treatment success stratified to the proposed lesion characteristics.

In summary, the new CFA lesion classification proposed by Rabellino et al. should be applied to all upcoming 
studies for improving study comparability and identification of potential predictors of acute and long-term treatment success for each individual treatment modality.

\section{Acknowledgements None.}

Funding Open Access funding enabled and organized by Projekt DEAL. This commentary was not supported by any funding.

\section{Declarations}

Conflict of interest Honoraria received from Abbott Vascular, BIBA Medical, Biotronik, Boston Scientific Corp., Cook Medical, Efemoral, Gore \& Associates, Medtronic, Philips-Spectranetics, Shockwave, Veryan. Consulted for Boston Scientific Corp., CSI, Gore \& Associates, Medtronic, Veryan, Philips-Intact Vascular, Shockwave, Bayer, Vesper Medical, VentureMed, ANT. Institutional Grants for research, clinical trial, or drug studies received from Bard Peripheral Vascular, Veryan, Biotronik, Cook Medical, Gore \& Associates, Medtronic, Philips, Terumo, TriReme, Shockwave, Med Alliance, Intact Vascular, B. Braun; CSI, Boston Scientific, University of Jena, Pluristem, Philips, PQ Bypass. Common stock: QT Medical.

Ethical Approval For this commentary, formal consent is not required.

Informed Consent For this commentary, informed consent is not required.

Consent for Publication For this commentary, consent for publication is not required.

Open Access This article is licensed under a Creative Commons Attribution 4.0 International License, which permits use, sharing, adaptation, distribution and reproduction in any medium or format, as long as you give appropriate credit to the original author(s) and the source, provide a link to the Creative Commons licence, and indicate if changes were made. The images or other third party material in this article are included in the article's Creative Commons licence, unless indicated otherwise in a credit line to the material. If material is not included in the article's Creative Commons licence and your intended use is not permitted by statutory regulation or exceeds the permitted use, you will need to obtain permission directly from the copyright holder. To view a copy of this licence, visit http://creativecommons. org/licenses/by/4.0/.

\section{References}

1. Gouëffic Y, Della Schiava N, Thaveau F, et al. Stenting or Surgery for De Novo Common Femoral Artery Stenosis. JACC Cardiovasc Interv. 2017;10(13):1344-54. https://doi.org/10.1016/j.jcin.2017. 03.046.

2. Bonvini RF, Rastan A, Sixt S, Noory E, Schwarz T, Frank U, et al. Endovascular treatment of common femoral artery disease: medium-term outcomes of 360 consecutive procedures. J Am Coll Cardiol. 2011;58(8):792-8.

3. Azéma L, Davaine JM, Guyomarch B, Chaillou P, Costargent A, Patra P, et al. Endovascular repair of common femoral artery and concomitant arterial lesions. Eur J Vasc Endovasc Surg. 2011;41(6):787-93. https://doi.org/10.1016/j.ejvs.2011.02.025 (Epub 2011 Mar 24).

4. Rabellino M, Valle Raleigh J, Chiabrando JG et al. Novel common femoral artery lesion classification in patients undergoing endovascular revascularization. Cardiovasc Intervent Radiol. 2021. https:// doi.org/10.1007/s00270-021-03011-z.

Publisher's Note Springer Nature remains neutral with regard to jurisdictional claims in published maps and institutional affiliations. 